\title{
Integrating population genetic structure, microbiome, and pathogens presence data in Dermacentor variabilis
}

\author{
Paula Lado $^{\text {Corresp., } 1}$, Bo Luan ${ }^{2}$, Michelle EJ Allerdice ${ }^{3}$, Christopher D Paddock $^{3}$, Sandor E Karpathy ${ }^{3}$, Hans Klompen \\ ${ }^{1}$ Evolution, Ecology, and Organismal Biology, The Ohio State University, Columbus, Ohio, United States \\ 2 Statistics, The Ohio State University, Columbus, Ohio, United States \\ 3 Rickettsial Zoonoses Branch, Centers for Disease Control and Prevention, Atlanta, Georgia, United States \\ Corresponding Author: Paula Lado \\ Email address: ladohenaise.1@osu.edu
}

Tick-borne diseases (TBDs) continue to emerge and re-emerge in several regions of the world, highlighting the need for novel and effective control strategies. The development of effective strategies requires a better understanding of TBDs ecology, and given the complexity of these systems, interdisciplinary approaches are required. In recent years, the microbiome of vectors has received much attention, mainly because associations between native microbes and pathogens may provide a new promising path towards the disruption of pathogen transmission. However, we still do not fully understand how host genetics and environmental factors interact to shape the microbiome of organisms, or how pathogenic microorganisms affect the microbiome and vice versa. The integration of different lines of evidence may be the key to improve our understanding of TBDs ecology. In that context, we generated microbiome and pathogen presence data for Dermacentor variabilis, and integrated those data sets with population genetic data, and metadata for the same individual tick specimens. Clustering and multivariate statistical methods were used to combine, analyze, and visualize data sets. Interpretation of the results is challenging, likely due to the low levels of genetic diversity and the high abundance of a few taxa in the microbiome. Francisella was dominant in almost all ticks, regardless of geography or sex. Nevertheless, our results showed that, overall, ticks from different geographic regions differ in their microbiome composition. Additionally, DNA of Rickettsia rhipicephali, R. montanensis, $R$. bellii, and Anaplasma spp., was detected in D. variabilis specimens. This is the first study that successfully generated microbiome, population genetics, and pathogen presence data from the same individual ticks, and that attempted to combine the different lines of evidence. The approaches and pre-processing steps used can be applied to a variety of taxa, and help better understand ecological processes in biological systems. 
1 Integrating population genetic structure, microbiome, and pathogens presence data in

2

3

4 5

6

7

8 9

\section{Dermacentor variabilis}

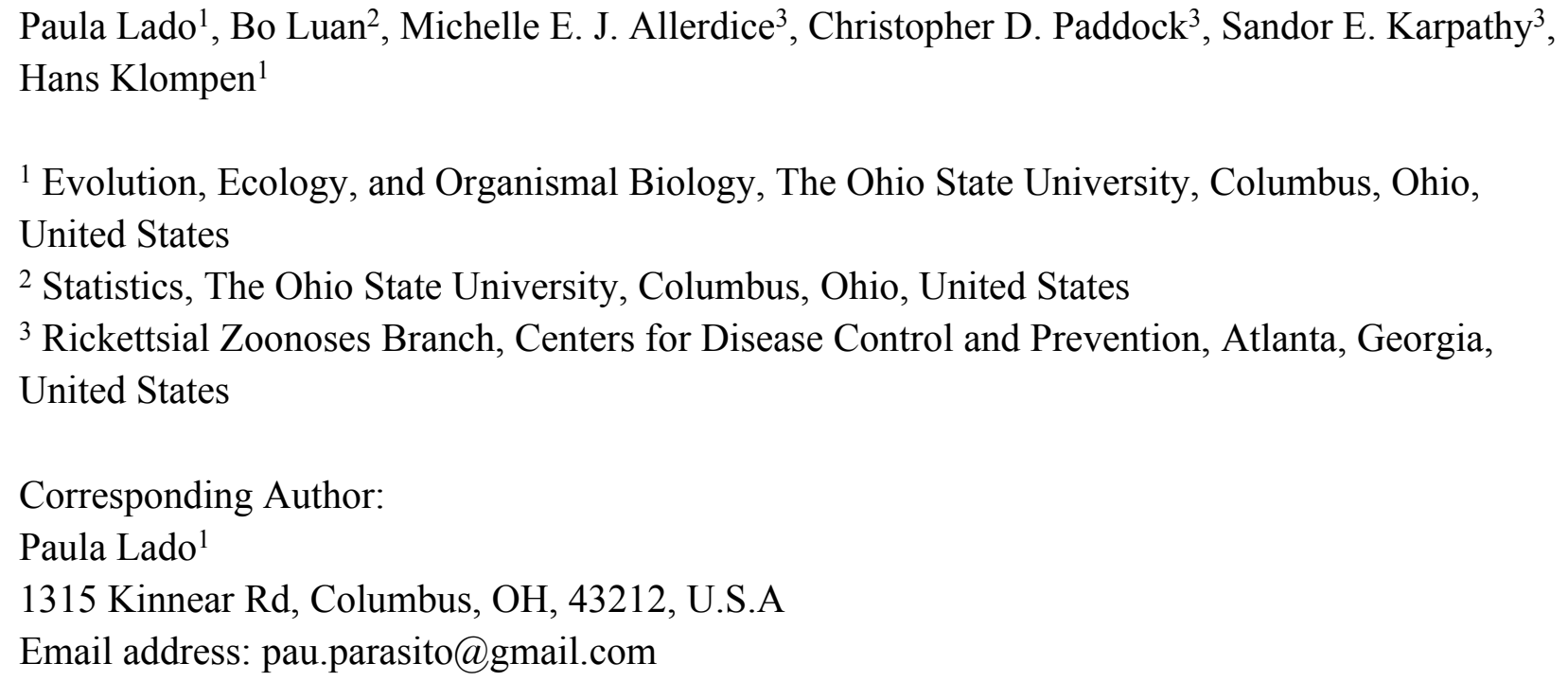

\section{ABSTRACT}

Tick-borne diseases (TBDs) continue to emerge and re-emerge in several regions of the world, highlighting the need for novel and effective control strategies. The development of effective strategies requires a better understanding of TBDs ecology, and given the complexity of these systems, interdisciplinary approaches are required. In recent years, the microbiome of vectors has received much attention, mainly because associations between native microbes and pathogens may provide a new promising path towards the disruption of pathogen transmission. However, we still do not fully understand how host genetics and environmental factors interact to shape the microbiome of organisms, or how pathogenic microorganisms affect the microbiome and vice versa. The integration of different lines of evidence may be the key to improve our understanding of TBDs ecology. In that context, we generated microbiome and pathogen presence data for Dermacentor variabilis, and integrated those data sets with 
30 population genetic data, and metadata for the same individual tick specimens. Clustering and

31 multivariate statistical methods were used to combine, analyze, and visualize data sets.

32 Interpretation of the results is challenging, likely due to the low levels of genetic diversity and

33 the high abundance of a few taxa in the microbiome. Francisella was dominant in almost all

34 ticks, regardless of geography or sex. Nevertheless, our results showed that, overall, ticks from

35 different geographic regions differ in their microbiome composition. Additionally, DNA of

36 Rickettsia rhipicephali, R. montanensis, R. bellii, and Anaplasma spp., was detected in D.

37 variabilis specimens. This is the first study that successfully generated microbiome, population

38 genetics, and pathogen presence data from the same individual ticks, and that attempted to

39 combine the different lines of evidence. The approaches and pre-processing steps used can be

40 applied to a variety of taxa, and help better understand ecological processes in biological

41 systems.

\section{INTRODUCTION}

As vector-borne diseases continue to emerge and re-emerge in several regions of the world, there is an urgent need for a better understanding of their ecology, including the tripartite pathogen-vector-host relationship, to ultimately develop effective control strategies. Ticks represent an increasing threat to One Health given the range expansion of some species, and the frequent identification of new tick-borne pathogens (Paddock et al. 2016; Eisen et al. 2017). Due to the complex nature of diseases transmitted by ticks, their control requires interdisciplinary studies and collaboration (Murgia et al. 2019). vectors (mostly mosquitoes and ticks) has received much attention. The main reason is that 
53 associations between native microbes and pathogens may provide a new promising path towards

54 the disruption of pathogen transmission (Narasimhan \& Fikrig 2015; Bonnet et al. 2017). In the

55 case of ticks, microbes can be acquired from the environment (e.g through the spiracles),

56 horizontally (from the host, during blood feeding), or vertically (maternally inherited).

57 Relationships between the tick and a particular microbe or set of microbes can range from

58 mutualistic to parasitic (Casadevall et al. 2011; Bonnet et al. 2017). Microbes can affect tick

59 fitness, vector competence, and pathogen transmission (Bonnet et al. 2017; Budachetri et al.

60 2018). For example, a few maternally inherited microbes have been proposed as primary

61 symbionts necessary for tick success by providing vitamins that are lacking in blood (Smith et al.

62 2015b; Duron et al. 2017; Guizzo et al. 2017). Without these microbes, the ticks' fitness is

63 negatively impacted (Zhong et al. 2007). Other symbionts interact with pathogenic

64 microorganisms, either positively or negatively. High proportions of the endosymbiotic

65 Rickettsia bellii inhibit the transmission of the pathogenic Anaplasma marginale (Gall et al.

66 2016), and $R$. parkeri is likely excluded from A. maculatum by Candidatus Rickettsia andeanae

67 (Paddock et al. 2015), presumably through a phenomenon known as interference. On the other

68 hand, cases of facilitation have also been reported; such as a positive relationship between the

69 proportion of the microbiome occupied by Francisella endosymbiont (FLE) and the infection

70 level of $F$. novicida (Gall et al., 2016).

71 Even though a considerable amount of information and knowledge has been generated

72 during the last decade or so, microbiome research on ticks is still in its infancy. For example, we

73 still do not fully understand how host genetics and environmental factors interact to shape the

74 microbiome of organisms (Spor et al. 2011; Goodrich et al. 2014; Steury et al. 2019), or how

75 pathogenic microorganisms affect the microbiome and vice versa. Integration of different lines 
76 of evidence may be the key to improve our understanding of TBDs ecology. As highlighted by

77 Griffiths et al. (2018), research exploring the links between, for example, vector genetics,

78 microbiome composition and structure, and pathogen susceptibility may enable a better

79 understanding of the factors governing disease in vulnerable populations. Unfortunately, to the

80 best of our knowledge, there are no such studies on ticks. There are a few studies based on

81 vertebrate taxa that have attempted to incorporate genomic and microbial data with data on

82 environmental variation (Fietz et al. 2018; Griffiths et al. 2018; Steury et al. 2019). Overall, these

83 studies have shown that genetically divergent host populations, exhibited more divergent

84 microbiomes (Smith et al. 2015a; Griffiths et al. 2018; Steury et al. 2019). In amphibians,

85 Griffiths et al. (2018) found that the genetic distance among hosts was correlated with microbial

86 community dissimilarity when controlling for geographic distance. Nevertheless, the same study

87 showed that at the site-level the microbiome did not mirror the host population genetic structure.

88 Steury et al. (2019) investigated populations of Threespine Stickleback and concluded that the

89 microbiome composition was better predicted by fish population genetic divergence than by

90 geographic distance and environment. It is worth noting that the global trends appear to be driven

91 by a subset of the microbiome. In other words, the influence of host genetic factors on the

92 microbiome composition depends on the bacterial taxa in question; host genetics may affect the

93 presence of some microbes, but presence of others may be better explained by, for example, the

94 environment (Fietz et al. 2018). In the same way, differences between populations could be the

95 result of differences in the relative abundance of a small subset of microorganisms (Steury et al.

96 2019).

97 The vertebrate results suggest that integrating microbiome and population genetic data

98 may lead to better understanding of the ecology of tick-borne diseases. Do ticks from different 
99 genetic clusters harbor a different microbiome? Do infected ticks belong to a specific genetic

100 cluster? Is the microbiome of infected ticks different from that of non-infected ticks? To start

101 answering these questions, it is paramount to first generate high quality data, and then to develop

102 an appropriated framework to integrate the different lines of evidence. This exploratory study

103 focuses on Dermacentor variabilis (Say), a North American tick species that commonly bites

104 humans, and that historically has been implicated in the transmission of several pathogens (Hecht

105 et al. 2019). Dermacentor variabilis is also one of the most widely distributed ticks in the United

106 States (Eisen et al. 2017), one for which population genetic data was already available. During

107 this investigation, we first generated high-quality microbiome data and determined the

108 presence/absence of several pathogens. Next, we combined those data sets with an existing

109 population genetics data set for D. variabilis (see Lado et al. 2019). All three pieces of data

110 (microbiome, pathogens, and population genetics) were derived from each of the individual ticks

111 included in this study. The goal of this preliminary study was to integrate the different lines of

112 evidence for D. variabilis ticks using clustering methods and multivariate statistics to identify

113 trends and patterns of variation. Our expectation was that D. variabilis ticks that are genetically

114 more alike (i.e. that belong to the same genetic cluster), will also be more alike at the

115 microbiome level. Additionally, we hypothesized that infected and non-infected ticks would

116 have a subset of microbes in their microbiome that are characteristic for each category.

\section{METHODS}

120 Tick samples 
The tick specimens employed in this study are the same individual ticks from Lado et al.

122 (2019), with the exception of two specimens for which we did not have enough genetic material.

123 The sample includes 64 adult D. variabilis collected from California $(n=3)$, Georgia $(n=2)$,

124 Indiana $(n=3)$, Maryland $(n=4)$, Maine $(n=3)$, Minnesota $(n=5)$, North Dakota $(n=1)$, Ohio

$125(n=31)$, Oklahoma $(n=5)$, Tennessee $(n=5)$, Virginia $(n=1)$, and Washington $(n=1)$ (Fig. 1). All

126 specimens were wild caught by dragging a $1 \mathrm{~m} \times 0.8 \mathrm{~m}$ cloth thorugh the vegetation, and

127 preserved in $95 \%$ ethanol. We collected ticks from vegetation (unfed) to minimize the amount of

128 genetic material from the host. Collection data for the specimens used in this study can be

129 accessed through the Ohio State Acarology Collection (OSAL) online database

130 (https://acarology.osu.edu). Metro Parks permitted collection of tick species throughout the

131 Metro Parks. The Division of State Parks (DNR), Indiana Department of Natural Resources

132 authorized tick collection at Brown County State Park, Clifty Falls State Park, O'Bannon Woods

133 State Park, Monroe Lake, Splinter Ridge Fish and Wildlife Area, Clark State Forest, and

134 Ferdinand State Forest.

\section{DNA extraction}

Before DNA extraction the ticks were surface sterilized following Lado et al. (2018);

138 specifically, the washes consist on following this procedure twice: commercial bleach $3 \%$ for

139 one minute, washed in distilled water, and 95\% ethanol for another minute. DNA extractions of

140 individual tick specimens were performed using the QIAgen Blood and Tissue kit following the

141 manufacturers' instructions, with one exception as in Lado et al. (2018): during the incubation in

142 ATL buffer the posterior-lateral part of the tick idiosoma was cut with a scalpel to allow a better

143 penetration to the buffer to the tick's tissues. The cuticle of all ticks was recovered and kept as 
144 voucher. OSAL accession numbers are listed in Table 1. Genomic DNA was quantified using a

145 Qubit 3.0 fluorometer; and then aliquoted and kept in the freezer until used.

146

147 Population genetic data

148 For the integrative portion of the analysis, we employed all genetic data generated

149 previously, and followed the genetic clusters nomenclature from Lado et al. (2019). Details on

150 how the population genetics data set was generated and analyzed can be found elsewhere (Lado

151 et al. 2019). In brief, ticks were assigned to three different genetic clusters according to their

152 population genetic structure: a generally "western cluster" $(n=4)$, an "eastern cluster" $(n=51)$,

153 and a "northern cluster" $(n=9)$. This clusters usually correspond to the locations where ticks were

154 collected.

155

156 Detection of Rickettsiales through PCR

157 PCR screening

158 Tick DNAs were tested by real-time PCR to detect three genera that include pathogenic

159 microorganisms: Rickettsia, Anaplasma, and Ehrlichia. All ticks were initially screened using

160 two real-time assays: 1) a TaqMan Pan-Rickettsia assay, which amplifies a portion of the 23S

161 gene for all Rickettsia species using primers PanR8-F and PanR8-R (Kato et al. 2013), and 2) an

162 EvaGreen Anaplasmataceae assay that targets a portion of the $16 \mathrm{~S}$ gene using primers

163 ECHSYBR-F and ECHSYBR-R, amplifying all Anaplasma and Ehrlichia species (Li et al.

164 2002).

165 Positive tick samples for Pan-Rickettsia (23S real-time assay) where further screened to

166 identify the Rickettsia species present. Samples were subjected to conventional semi-nested PCR 
167 targeting the ompA gene of all spotted fever group Rickettsia (Regnery et al. 1991; Eremeeva et

168 al. 2006). PCRs were performed using $1 \mu \mathrm{M}$ of each primer $(\operatorname{Rr} 190.70, \operatorname{Rr} 190.602, \operatorname{Rr} 190.701)$,

$16910 \mu \mathrm{L}$ of Taq PCR Master Mix (QIAGEN), $2 \mu \mathrm{L}$ of sample DNA in the primary reaction or $2 \mu \mathrm{l}$

170 of the primary reaction product in the secondary reaction, and water to bring the final reaction

171 volume to $20 \mu \mathrm{l}$. Positive samples were processed as described below to identify species present.

172 We also performed a $R$. bellii specific TaqMan assay targeting gltA, the citrate synthase gene

173 (Hecht et al. 2016) for all positive samples for Pan-Rickettsia.

174 All real-time PCRs were performed in duplicate on a BioRad CFX 96 thermal cycler

175 using $4 \mu \mathrm{L}$ of template DNA in a final reaction volume of $25 \mu \mathrm{L}$ for the Pan-Rickettsia and 20

$176 \mu \mathrm{L}$ for both the Anaplasmataceae and $R$. bellii-specific assays. We considered samples positive if

177 one of the duplicates had a cycle threshold $(\mathrm{Ct})<40$. Two sets of negative controls and one set of

178 positive controls were included on each plate, where water was used as the negative non-

179 template control and DNA from cultured R. rickettsii, E. canis, or an R. bellii plasmid were used

180 as positive controls, depending on the assay (Hecht et al. 2019).

181 Amplicon purification and sequencing

182 Amplicons from ompA semi-nested PCR were visualized on 1.5\% agarose gels using

183 ethidium bromide. Amplicons were extracted and purified using the Promega Wizard SV Gel

184 and PCR Clean-up System (Promega, Madison, WI). Products were bidirectionally sequenced

185 using a BigDye Terminator v3.1 kit on an ABI 3500 genetic analyzer (Applied BioSystems,

186 Carlsbad, CA) and assembled using Geneious version 7.0.4. (http:// geneious.com, Kearse et al.

187 2012). The nucleotide BLAST tool of the NCBI GenBank database was employed to compare

188 the amplicon sequences to those sequences available in the database. Positive amplicons from the 189 Anaplasmataceae assay were also sequenced following the above-mentioned procedure. 


\section{Microbiome}

192 16SrDNA library preparation and amplicon sequencing

193 Genomic DNA samples, along with two negative controls, were taken to a final

194 concentration of $10 \mathrm{ng} / \mu 1$, and shipped to Argonne National Laboratory for library preparation

195 and sequencing following standard procedures. The two negative controls correspond to a

196 extraction blank and library blank sensu Kim et al. 2017. The primer pair 515F/926R (Walters et

197 al. 2016) was employed to amplify the V4-V5 variable regions of the 16S rDNA gene, and then

198 the amplicons were sequenced on a MiSeq illumina platform, paired-end $251 \mathrm{bp}$ reads. The

199 obtained reads were demultiplexed using MiSeq Reporter.

200 Quality filtering, OTU picking, taxonomic assignments, and diversity calculations

201 Data were initially filtered as previously described in Lado et al. (2018). Specifically,

202 after demultiplexing, Cutadapt (Martin 2011) was used to do an initial quality filter of the reads

203 (threshold Q10), and to trim the adaptors if they were present in the filtered reads. Once the reads

204 passed the initial filters, the QIIME 1.9.1 (Caporaso et al. 2010) pipeline was employed to:

205 assemble the reads; cluster the reads (97\% threshold); and to assign taxonomy. Open reference

206 OTUs (operational taxonomic units) picking using uclust, and taxonomic assignment using the

207 Greengenes (DeSantis et al. 2006) and Silva132 (Quast et al. 2013) data bases. An alignment of

208 representative sequences was used as input to generate a tree in FastTree (Price et al. 2009); as

209 well as to construct OTU tables for each of the taxonomic level (i.e genus, family). OTUs

210 abundant in the negative controls and suspected as contaminants were removed in $\mathrm{R}$ using the

211 decontam package (Davis et al. 2018). 


\section{Statistical analysis: integrating individual data sets}

214 OTU tables were imported into R, and finer filtering was performed. First, we eliminated

215 all OTUs that appeared in no more than two samples. Then, with the reduced data, we further

216 eliminated OTUs with less than $0.5 \%$ relative abundance in all ticks. The dimensions of the data

217 were therefore substantially reduced. Metadata was then added to the tables, including

218 coordinates, sex, genetic cluster they belong to (following Lado et al. 2019), and the presence of:

219 Rickettsia, Anaplasma or Ehrlichia species for each tick specimen. Additionally, ticks were

220 assigned to three geographic regions following Lado et al. 2019: eastern, western, and northern.

221 Clustering approach

222

To determine how samples are related to one another when integrating all variables, a

223 hierarchical clustering approach was taken. In this analysis the variables included were the

224 filtered OTU table, tick ID, sex, genetic cluster, location (as coordinates), and presence of

225 Rickettsia or Anaplasmataceae agents. Hierarchical clustering was performed in R and the

226 number of clusters was set to three $(\mathrm{k}=3)$, which is the number of genetic clusters. We employed

227 Gower's distance (Gower 1971) as the measure of dissimilarity between samples because it is

228 compatible with mixed data types (quantitative, nominal, and binary variables). We visualized

229 the clustering results through the dendrogram generated by the hierarchy and demonstrated

230 sample organization in space by using the first two principal components of all the filtered OTUs

231 and location coordinates.

232 As a further exploration, we evaluated how well our clustering matched with the genetic

233 clusters by the concordance index (C-index), which is an internal validation measure of goodness

234 of matching. We also employed methods to determine the optimal number of clusters from the

235 data. This allowed the comparison between the predicted number of clusters $(\mathrm{k}=3)$, and the 
236 number of clusters determined inherently from the data set. For this purpose, two methods were

237 used: the average silhouette and the elbow method. The former method computes the average

238 silhouette of observations for different values of $k$. The optimal number of clusters is the one that

239 maximizes the average silhouette over a range of possible values for $\mathrm{k}$ (Kaufman \& Rousseeuw

240 1990). On the other hand, the elbow method looks at the total within-cluster sum of squares

241 (wss) as a function of the number of clusters. The optimal $k$ will be the smallest one such that

242 adding another cluster doesn't improve much better the total wss.

243 Ordination methods

244 Once the data was integrated, ordination methods were used to visualize the samples in

245 space. We used both non-metric multidimensional scaling (NMDS) and Principal coordinates

246 analyses (PCoA) to determine if there was any patterning within the data. Both techniques

247 reduce the high dimensional data into a two-dimensional representation. All analysis and graphs

248 were done in R, employing the phyloseq package (McMurdie \& Holmes 2013).

249 Test for significance for different variables

250 We used Welch's t-test to detect if the relative abundance of a specific species of

251 microorganism differed across host sex or between genetic clusters. Such tests were shown to be

252 flexible and robust even when samples sizes were unbalanced and group variances were unequal

253 (Delacre et al. 2017).

254 To compare microbial beta diversity across treatments (sex, genetic cluster, and region)

255 we calculated the distance between microbial communities by using two metrics. The first one,

256 Bray-Curtis distance, considers the relative abundance of bacteria, while the second, Jaccard's

257 distance (or dissimilarity index), measures differences in presence/absence of Bacteria. All

258 distances were calculated using the vegan package (Oksanen et al. 2019) in R. We then 
259 partitioned the matrices by treatment and performed permutational multivariate analysis of

260 variance (PERMANOVA) (Anderson 2001) to determine if beta diversity differed between

261 treatments. PERMANOVA calculations were performed using the adonis function in the vegan

262 package with 999 permutations. For those treatments that included more than two groups, a post

263 hoc test was performed to identify the pairs of groups between which the bulk of differences

264 occurred. For that purpose, we employed the paiwise.adonis function from the same package.

265

266

\section{RESULTS}

267

268

269

270

271

272

273

274

275

276

277

278

279

280

281

\section{Detection of Rickettsiales through PCR}

DNA belonging to the genus Rickettsia was detected in $15.6 \%(10 / 64)$ of the screened ticks including $R$. montanensis $(\mathrm{n}=6,9.3 \%) ;$. rhipicephali $(\mathrm{n}=2,3.1 \%)$; and $R$. bellii $(\mathrm{n}=2$, 3.1\%). Rickettsia bellii was detected only in specimens collected from the western U.S. Anaplasma DNA was found in a single tick (1.6\%), collected in Oklahoma. The DNA sequence obtained was identified as "Uncultured Anaplasma sp. clone 15-3642 16S ribosomal RNA gene, GenBank number MG429812". The sequence generated in this study was $100 \%$ identical to MG429812, and the query coverage was also $100 \%$, corresponding to bases 23 to 168 .

None of the ticks were infected with Ehrlichia species. A summary of PCR results and metadata for each tick specimen is presented in Table 2 .

\section{Microbiome}

Microbiome data were successfully generated for all ticks. However, some issues were encountered when attempting to merge forward and reverse reads (only a minimal fraction of the reads merged correctly). Because of that and given the large amount of data obtained, only 
282 forward reads (R1) were employed for all downstream analysis. It is possible that the difficulties

283 encountered when merging the reads is due to the primers pair selected, as other research groups

284 have faced similar issues with these primers for different taxa (Argonne National Lab personal

285 communication). The number of Illumina forward (R1) reads obtained was 2,131,680. After

286 quality filtering the number of reads retained was 2,129,331 with an average length of $251 \mathrm{bp}$.

287 The average number of sequences per sample was 32,795 (minimum $=9,331$; maximum $=$

288 44,533). The number of reads for the extraction blank was 2349, considerably lower than all

289 samples; and no reads were generated from the library blank.

290 A total of 41 orders of bacteria were identified (13 remaining after filtering), and three of them

291 were dominant: Rickettsiales, Legionellales, and Enterobacteriales (Fig. 2A). At the genus level,

29261 taxa were detected (Supplementary file 1). Five taxa were removed since their relative

293 abundance was greater in the negative control than in tick samples. Of the 56 genera remaining,

29436 were eliminated during the filtering steps according to minimum relative abundance and the

295 number of samples ( see Methods section for details). Thus, 20 genera were kept for downstream

296 analyses (Supplementary file 1). Three taxa were highly abundant at the genus level: Rickettsia

297 spp., Francisella spp., and Enterobacteriaceae “other". (Fig. 2B). Francisella spp. were present

298 in all ticks, with a relative abundance ranging from 0.4 to $100 \%$ (mean $=81 \%$, median $=98.5 \%)$.

299 Rickettsia was detected in 12 (18.8\%) of the ticks. In all cases where Rickettsia spp. had a

300 relative abundance of at least $0.5 \%$ by microbiome analysis, tick specimens were also PCR

301 positive, thus microbiome and PCR results are overall, congruent (Table 2). Rickettsiella, an

302 intracellular endosymbiont of arthropods (Leclerque 2008), was found in a tick from Ohio (1/64;

303 tick ID 119572D) with a relative abundance of $11 \%$. 
305 Statistical analysis: integrating individual data sets

306 Clustering approach

307 The dendrogram resulting from hierarchical clustering showed that the two most distinct

308 samples (clustering together) were from the same location and collection event: Fernald

309 Preserve, Hamilton Co., Ohio (110559-1 and 110559-2) (Fig. 3). The second clade grouped two

310 samples from CA, the two samples collected in Lake Co (115102A,B). The next pair of samples

311 corresponded to the two remaining samples from the west coast, a sample from Napa Co, CA

312 (115101C); and one from Whitman Co, WA (115105B). These two samples were closely

313 associated with a clade that included all Northern samples. Lastly, all Eastern samples clustered

314 together, without any internal grouping according to geography (Fig. 3).

315 No differences were evident upon visualization of community membership or structure. PCA did

316 not show any specific clustering pattern between samples (Supplementary file 2). In fact, most of

317 the ticks were clustered in a single aggregate. The ticks that clustered somewhat separated were

318 firstly the two that also appeared separated in the clustering analysis (110559-1 and 110559-2);

319 and a tick from Ohio, 119241-3. It is important to note that the first two principal components

320 explained only approximately $40 \%$ of the variance present in our sample.

321 As for $k$, the optimal number of clusters, silhouette method and elbow method suggested

$322 k=2$ and $k=4$ respectively (Supplementary file 3 ). Both values were close to the number of

323 genetic clusters, which was also the number of clusters we used in the analysis. The C-index

324 between the predicted clusters and genetic clusters was 0.61 , indicating some weak but

325 nonnegligible consistency between these two pairs.

326 Ordination methods

327 When visualizing the samples in the space through NMDS (stress value 0.064), the 
328 samples were spread out without any specific pattern (Fig. 4A). In PCoA with Gower's distance

329 as metric, most samples appeared in a single tight aggregate, while a few others dispersed

330 randomly along the axes (Fig. 4B).

331 Test for significance for different variables

332 The number of bacterial genera found in ticks belonging to the Eastern genetic cluster

333 was higher than that of the Western, and Northern clusters. The genus Francisella was dominant

334 in almost all the ticks, regardless of geography. Its relative abundance did not differ significantly

335 between males and females ( $\mathrm{p}$-val 0.8); nor between ticks belonging to different genetic clusters

336 (Eastern vs Western p-val 0.57, Eastern vs Northern p-val 0.96, Northern vs Western p-val 0.60)

337 (Fig. 5). The relative abundance of this genus was also not significantly different between

338 infected and uninfected ticks (p-val 0.14), although it was generally lower in infected ticks.

339 Infected ticks were defined as those that were PCR positive for either Anaplasma or Rickettsia

340 species.

341 Rickettsia relative abundance did not significantly differ between males and females (p-

342 val 0.44), nor between ticks belonging to different genetic clusters (Northern vs. Eastern p-val =

343 0.26; Northern vs. Western p-val $=0.72$; and Eastern vs. Western p-val $=0.45)($ Fig. 5).

344 Rickettsia rhipicephali and Anaplasma spp. were present only in ticks belonging to the Eastern

345 genetic cluster, and R. montanensis was present in ticks belonging to the Northern and Eastern

346 clusters. Rickettsia bellii was found infecting ticks from the Western and Northern genetic

347 clusters, although of these ticks were collected in the Western region (Fig 6). One of the ticks

348 positive for this species was $115101 \mathrm{C}$, a tick collected in California but with the genetic make-up

349 corresponding to Northern ticks.

350 Overall, microbial beta diversity differed between regions ( $\mathrm{p}$-val $<0.05)$, but not between 
351 sexes $(p=0.63)$ when using Bray-Curtis distance. Post hoc tests indicated that ticks collected in

352 the Western region harbored a different microbiome from those collected in the Eastern or

353 Northern regions $(\mathrm{p}<0.05)$. In the case of presence/absence of Bacteria (Jaccard distance), the

354 results were the same. A significant difference between ticks collected from different regions

355 was detected $(\mathrm{p}<0.05)$, and the post hoc tests showed that the differences were significant

356 between the Western and both the Eastern and Northern regions.

357 The Adonis test for genetic clusters showed that there were differences $(\mathrm{p}<0.05)$ only

358 when considering relative abundance of bacterial taxa (Bray-Curtis distance). When the pairwise

359 Adonis test was performed to determine what genetic clusters differed in microbiome structure

$360(\mathrm{p}<0.05)$ between Eastern and Western genetic clusters, but its correction was not ( $\mathrm{plFDR}$

361 0.096). Eastern and Western clades are genetically, the two more distant (Lado et al. 2019).

362

\section{DISCUSSION}

This work corresponds, to the best of our knowledge, to the first attempt in tick research

to integrate population genetic, microbiome, and pathogens presence data to better understand the ecology of TBDs. To optimize comparisons, it was our goal to generate those three pieces of data for each of the individual ticks included in the data set. We show that this is possible, even when techniques based on next generation sequencing require high quantities of high quality DNA.

We developed an effective pre-processing and processing procedure for researchers interested in microbiomes of small organisms, or parts of organisms with limited amounts of

372 genetic material. This was motivated by a need for consensus regarding the pre-processing of

373 ticks for generating microbiome data. For example, it is likely that many of the incongruencies 
374 between microbiome studies arise from differences in approaches to decontamination of the

375 ticks' surface. Several studies in the literature performed surface sterilization of the ticks before

376 DNA extraction (e.g. Lado et al. 2018; Trout Fryxell and DeBruyn 2016), whereas others did not

377 (e.g. Clow et al. 2018); and the differences in bacterial communities may be a reflection of

378 extensive environmental "contamination" in the latter. Even between studies that "washed" the

379 ticks, comparisons need to be done cautiously, since not all sterilization methods are equally

380 effective. The incorporation of bleach appears necessary for effective decontamination (Binetruy

381 et al. 2019). Increased consistency in results of microbiome studies of ticks requires some

382 community consensus related to the generation and analysis of microbiome data. This is

383 especially important for non-model organisms such as ticks, given the "noise" (Alpha and Beta

384 diversity inflation) generated by the presence of environmental microorganisms.

385 Consistent with most other studies of tick microbiomes (Van Treuren et al. 2015; Gall et al.

386 2016; Chicana et al. 2019), our analysis showed that the microbiome of many species is heavily

387 dominated by a few genera, while the microbiomes of others, such as Ixodes angustus (Chicana

388 et al. 2019) and Haemaphysalis lemuris (Lado et al. 2018), are more diverse. The microbiome of

389 D. variabilis is dominated by Francisella (Chicana et al. 2019; Travanty et al. 2019; current

390 study). Clow et al. (2018) presented, to some extent, opposing results (far higher microbial

391 diversity), but they analyzed only 9 specimens, and the ticks were not surface sterilized. These

392 results casts some doubt on the concept of a "core microbiome". For example, Chicana et al.

393 (2019) report a D. variabilis core microbiome as composed by Francisella, Sphingomonas, and

394 Methylobacterium. On the other hand, Travanty et al. (2019) reported a D. variabilis core

395 microbiome that includes Francisella spp., Sphingomonas spp., Delftia spp.,

396 and Hymenobacter spp. Our study recovered all of these taxa but only Fransicella spp. is nearly 
397 universally present. Francisella has been well established as endosymbiont and dominant in $D$.

398 variabilis, as well as other Dermacentor species. All other taxa reported as "core microbiome"

399 are not consistent across studies (e.g. Chicana et al. 2019; Clow et al. 2018; Rynkiewicz et al.

400 2015; Travanty et al. 2019). Thus, the true "core" of taxa overlapping across studies, can be

401 reduced to Francisella only. The concept of "core microbiome" is generally used in microbiome

402 research to refer to a suite of microbes, and not to refer to only one taxon. The utility of the

403 concept of "core microbiome" for D. variabilis is therefore unclear.

404 Recent studies focusing on the pathogen transmission by D. variabis and on its role as

405 vector of human diseases have consistently reported low prevalence of infections with known

406 pathogenic microorganisms. For example, $R$. rickettsii, is usually present in $\sim 1 \%$ of the ticks

407 analyzed e.g. (Hecht et al. 2019). The results obtained in the present study are consistent with

408 these literature results, and show an overall Rickettsia spp. prevalence of $17.2 \%$, including $R$.

409 montanensis, $R$. bellii, and $R$. amblyommatis infections. Rickettsia rickettsii DNA was not found

410 in any of the ticks analyzed. We detected DNA of Anaplasma spp. in one tick sample collected

411 in Oklahoma. Its DNA sequence matched 100\% with GenBank sequences submitted by

412 researchers at the CDC Fort Collins, who isolated the agent from a human blood sample. No

413 additional information is available in the literature about this case. This study comprises the first

414 report of that specific bacterial agent in D. variabilis. However, an Anaplasma spp. bovis-like

415 agent has been previously reported in D. andersoni ticks from Canada (Dergousoff \& Chilton

416 2011; Chilton et al. 2018). Unfortunately, the region of 16S amplified differs between our study

417 and that of D. andersoni (Dergousoff \& Chilton, 2011), making it difficult to determine if the

418 sequences correspond to the same specific agent. Regardless, it appears that uncharacterized

419 Anaplasma agents are circulating in nature (ticks and humans), and their characterization, 
420 together with the determination of whether or not they are capable of causing disease in humans

421 should be further explored. Furthermore, due to the finding of this agent's DNA in a $D$.

422 variabilis sample in this study, the role of $D$. variabilis as potential vector should be considered 423 and further explored.

424 With respect to the primary goal of this preliminary study, the integration of the different

425 types of data was done successfully, although the interpretation of the results is somewhat

426 challenging. The latter problem is most likely caused by to the low levels of genetic diversity and

427 moderate levels of population structure (see details in Lado et al. 2019), and a microbiome

428 highly dominated by a few taxa. As a result, ordination methods failed to show patterns of

variation across different groups. Nonetheless, the dendrogram resulting from the clustering

430 analysis, in which all lines of evidence and geographic location were considered, was largely

431 congruent with the observations at the population genetic level. Thus, the addition of

432 microbiome, pathogen presence, ticks sex, and geographic location led to conclusions that are, in 433 general, consistent with ticks genetics. All Eastern samples clustered together (with the exception

434 of two samples from Hamilton $\mathrm{Co}, \mathrm{OH}$ ), and separated from both Western and Northern

435 samples. The distinctiveness and separation of those two samples from Hamilton Co, OH reflects

436 their distinctiveness at the microbiome level (Fig. 2). The fact that both samples appeared very

437 similar to all other Eastern samples at the population genetic level (Lado et al. 2019), supports

438 that hypothesis. It is possible that these two specimens were not well surface sterilized, or that

439 the environment at that collection location is different.

440 The higher number of bacterial genera found in ticks belonging to the Eastern genetic

441 cluster when compared to either Northern or Western ticks, is likely a product of the higher

442 number of ticks analyzed, and the greater geographic area covered. Even though in the broader 
443 sense the microbiome is not diverse (dominated by three genera), it differed between the three

444 main geographic regions: Eastern, Western, and Northern. Statistical results looking at the beta

445 diversity between genetic clusters are less clear: initial test demonstrated a difference in the

446 microbial communities between genetic clusters, but pairwise tests with their corresponding

447 corrections failed to reach the same result. This may be a result of one of the study's limitations,

448 the number of samples. Thus, these findings should be further explored using more samples, and

449 more samples per genetic cluster. From our preliminary analyses, geography explains the

450 differences in the microbial communities better than host genetics. This could be the result of

451 certain microorganisms occurring only in certain geographic areas.

452 Despite challenges arising during the interpretation of the results, the integration of lines

453 of evidence and metadata, revealed that Eastern ticks can be separated from Northern and

454 Western ticks. It can also be noted that some Rickettsia species were associated with a certain

455 geographic area. For example, $R$. bellii was found only in samples collected in CA, a finding

456 consistent with that reported in Hecht et. al. (2019). It seems possible that $R$. bellii is more

457 common along the west coast, although more samples should be analyzed to confirm this. And

458 while the two $R$. bellii positive tick samples were collected in CA, one of them (ID 115101C) did

459 not belong to the Western genetic cluster; it belonged to the Northern cluster. From the host

460 genetics perspective, this tick is more similar to ticks from Northern locations; whereas from the

461 rickettsial agents perspective, it is more similar to Western samples. Looking at the microbiome

462 composition, this tick could belong to any geographic region. This underscores the value of

463 integrating different types of data when thinking about disease ecology.

464 The approaches taken during this study, both to generate and analyze data, can be applied

465 to a wide variety of taxa, and groups of organisms. It is likely that in organisms with a higher 
466 level of variation, the clustering methods will be more informative, and their interpretation more

467 straightforward. Therefore, the approaches used herein have potential, and could greatly improve

468 future studies looking at different aspects of diseases ecology. In the particular case of $D$.

469 variabilis, the approaches used herein failed to detect clear tendencies or patterns. This was

470 likely due to the low levels of variation (both in the microbiome and population genetics), and

471 the very low prevalence of pathogenic microorganisms.

472

473 CONCLUSIONS

474 In conclusion, this is the first study that successfully generated microbiome, population 475 genetics, and pathogens presence data for the same individual ticks. General methodologies and 476 pre-processing steps are replicable, and applicable to different groups of organisms across the 477 tree of life. This work also comprises, at present, one of the few studies aiming at integrating 478 population genetics and microbiome data to better understand ecological processes and disease, 479 and it is the first one to do so for ticks. The integration of different lines of evidence allows a 480 more holistic approach; and clustering and ordination methods are very helpful to summarize and 481 visualize the results. Finally, this study comprises the first report of "Uncultured Anaplasma sp. 482 clone $15-3642$ " in $D$. variabilis. This agent was previously isolated from human blood, and it is 483 important to determine if it is capable of causing disease in humans, and if so, the role of 484 Dermacentor ticks as potential vectors.

\section{ACKNOWLEDGEMENTS}

We are grateful to M. Yoshimizu, A. Donohue, B. Ryan, G. Keeney, C. Nelson, L. Beati,

L. Durden, C. Lubekzyk, D. Neitzel, G. Hickling, and B. Pagac who provided some of the tick 
489 specimens used in this study. We also thank The National Ecological Observatory Network, a

490 program sponsored by the National Science Foundation and operated under cooperative

491 agreement by Battelle Memorial Institute, for providing some specimens used in this work. We

492 thank the Ohio Supercomputer Center for computing resources (allocation grant PAS1306), and

493 two anonymous reviewers for their constructive comments, which helped improving earlier

494 versions of this manuscript. The findings described herein are those of the authors and do not

495 necessarily represent the official position of the United States Department of Health and Human

496 Services.

497

498

\section{REFERENCES}

499

500

501

502

Anderson MJ. 2001. A new method for non-parametric multivariate analysis of variance. Austral Ecology 26:32-46.

Binetruy F, Dupraz M, Buysse M, Duron O. 2019. Surface sterilization methods impact measures of internal microbial diversity in ticks. Parasites and Vectors 12:268.

Bonnet SI, Binetruy F, Hernández-Jarguín AM, Duron O. 2017. The Tick Microbiome: why nonpathogenic microorganisms matter in tick biology and pathogen transmission. Frontiers in Cellular and Infection Microbiology 7:1-14.

Budachetri K, Kumar D, Crispell G, Beck C, Dasch G, Karim S. 2018. The tick endosymbiont Candidatus Midichloria mitochondrii and selenoproteins are essential for the growth of Rickettsia parkeri in the Gulf Coast tick vector. Microbiome 6:141.

Caporaso JG, Kuczynski J, Stombaugh J, Bittinger K, Bushman FD, Costello EK, Fierer N, Gonzalez Peña A, Goodrich JK, Gordon JI, Huttley GA, Kelley ST, Knights D, Koenig JE, Ley RE, Lozupone CA, McDonald D, Muegge BD, Pirrung M, Reeder J, Sevinsky JR, 
512 Turnbaugh PJ, Walters WA, Widmann J, Yatsunenko T, Zaneveld J, Knight R. 2010.

513 QIIME allows analysis of high-throughput community sequencing data. Nature Methods

$514 \quad 7: 335-336$.

515 Casadevall A, Fang FC, Pirofski L anne. 2011. Microbial virulence as an emergent property:

$516 \quad$ Consequences and opportunities. PLoS Pathogens 7:e1002136.

517 Chicana B, Couper LI, Kwan JY, Tahiraj E, Swei A. 2019. Comparative microbiome profiles of 518 sympatric tick species from the far-western United States. Insects 10:1-12.

519 Chilton NB, Dergousoff SJ, Lysyk TJ. 2018. Prevalence of Anaplasma bovis in Canadian

520 populations of the Rocky Mountain wood tick, Dermacentor andersoni. Ticks and Tick-

$521 \quad$ borne diseases 9:1528-1531.

522 Clow KM, Weese JS, Rousseau J, Jardine CM. 2018. Microbiota of field-collected Ixodes 523 scapularis and Dermacentor variabilis from eastern and southern Ontario, Canada. Ticks $524 \quad$ and Tick-borne Diseases 9:235-244.

525 Davis NM, Proctor D, Holmes SP, Relman DA, Callahan BJ. 2018. Simple statistical

526 identification and removal of contaminants sequences in marker-gene and metagenomics

527 data. Microbiome 6:226.

528 Delacre M, Lakens D, Leys C. 2017. Why psychologists should by default use welch's t-Test 529 instead of student's t-Test. International Review of Social Psychology 30:92-101.

530 Dergousoff SJ, Chilton NB. 2011. Novel genotypes of Anaplasma bovis, "Candidatus

531 Midichloria” sp. and Ignatzschineria sp. in the Rocky Mountain wood tick, Dermacentor

532 andersoni. Veterinary Microbiology 12;150(1-2):100-106.

533 DeSantis TZ, Hugenholtz P, Larsen N, Rojas M, Brodie EL, Keller K, Huber T, Dalevi D, Hu P, 534 Andersen GL. 2006. Greengenes, a chimera-checked 16S rRNA gene database and 
workbench compatible with ARB. Applied and environmental microbiology 72:5069-72.

536

537

538

539

540

541

542

543

544

545

546

547

548

549

550

551

552

553

554

555

556

557

Duron O, Binetruy F, Noël V, Cremaschi J, McCoy KD, Arnathau C, Plantard O, Goolsby J, Pérez de León AA, Heylen DJA, Van Oosten AR, Gottlieb Y, Baneth G, Guglielmone AA7, Estrada-Peña A, Opara MN, Zenner L, Vavre F, Chevillon C. 2017. Evolutionary changes in symbiont community structure in ticks. Molecular Ecology 26:2905-2921.

Eisen RJ, Kugeler KJ, Eisen L, Beard CB, Paddock CD. 2017. Tick-borne zoonoses in the United States: Persistent and emerging threats to human health. ILAR Journal 58:319-335.

Eremeeva ME, Bosserman EA, Demma LJ, Zambrano ML, Blau DM, Dasch GA. 2006. Isolation and identification of Rickettsia massiliae from Rhipicephalus sanguineus ticks collected in Arizona. Applied and Environmental Microbiology 72:5569-5577.

Fietz K, Rye Hintze CO, Skovrind M, Kjærgaard Nielsen T, Limborg MT, Krag MA, Palsbøll PJ, Hestbjerg Hansen L, Rask Møller P, Gilbert MTP. 2018. Mind the gut: Genomic insights to population divergence and gut microbial composition of two marine keystone species. Microbiome 6:82.

Gall CA, Reif KE, Scoles GA, Mason KL, Mousel M, Noh SM, Brayton KA. 2016. The bacterial microbiome of Dermacentor andersoni ticks influences pathogen susceptibility. The ISME Journal 10:1846-1855.

Goodrich JK, Goodrich JK, Waters JL, Poole AC, Sutter JL, Koren O, Blekhman R, Beaumont M, Van Treuren W, Knight R, Bell JT, Spector TD, Clark AG, Ley RE. 2014. Human genetics shape the gut microbiome. Cell 159(4):789-799.

Gower JC. 1971. A General Coefficient of Similarity and Some of Its Properties. Biometrics 27:857-871.

Griffiths SM, Harrison XA, Weldon C, Wood MD, Pretorius A, Hopkins K, Fox G, Preziosi RF, 
Antwis RE. 2018. Genetic variability and ontogeny predict microbiome structure in a disease-challenged montane amphibian. ISME Journal 12:2506-2517.

560 Guizzo MG, Parizi LF, Nunes RD, Schama R, Albano RM, Tirloni L, Oldiges DP, Vieira RP,

561 Oliveira WHC, Leite MS, Gonzales SA, Farber M, Martins O, Vaz IDS Jr, Oliveira PL.

562 2017. A Coxiella mutualist symbiont is essential to the development of Rhipicephalus

563 microplus. Scientific Reports 7:17554.

564 Hecht JA, Allerdice MEJ, Dykstra EA, Mastel L, Eisen RJ, Johnson TL, Gaff HD, Varela-Stokes

565 AS, Goddard J, Pagac BB, Paddock CD, Karpathy SE. 2019. Multistate Survey of

566 American Dog Ticks (Dermacentor variabilis) for Rickettsia Species. Vector-Borne and

567 Zoonotic Diseases 19:652-657.

568 Hecht JA, Allerdice MEJ, Krawczak FS, Labruna MB, Paddock CD, Karpathy SE. 2016.

569 Development of a Rickettsia bellii-specific taq man assay targeting the citrate synthase 570 gene. Journal of Medical Entomology 53(6):1492-1495.

571 Kato CY, Chung IH, Robinson LK, Austin AL, Dasch GA, Massunga RF. 2013. Assessment of 572 real-time PCR assay for detection of Rickettsia spp. and Rickettsia rickettsii in banked 573 clinical samples. Journal of Clinical Microbiology 51:314-417.

574 Kaufman L, Rousseeuw P. 1990. Finding Groups in Data: An Introduction to Cluster Analysis. 575 John Wiley \& Sons, Inc.

576 Kearse M, Moir R, Wilson A, Stones-Havas S, Cheung M, Sturrock S, Buxton S, Cooper A, 577 Markowitz S, Duran C, Thierer T, Ashton B, Meintjes P, Drummond A. 2012. Geneious

578 Basic: An integrated and extendable desktop software platform for the organization and $579 \quad$ analysis of sequence data. Bioinformatics 28:1647-1649.

580 Kim D, Hofstaedter CE, Zhao C, Mattei L, Tanes C, Clarke E, Lauder A, Sherrill-Mix S, 
581 Chehoud C, Kelsen J, Conrad M, Collman RG, Baldassano R, Bushman FD, Bittinger K.

582 2017. Optimizing methods and dodging pitfalls in microbiome research. Microbiome 5:52.

583 Lado P, Cox C, Wideman K, Hernandez A, Klompen H. 2019. Population genetics of

584 Dermacentor variabilis Say 1821 (Ixodida: Ixodidae) in the United States Inferred from

585 ddRAD-seq SNP Markers. Annals of the Entomological Society of America 112:433-442.

586 Lado P, Qurollo B, Williams C, Junge R, Klompen H. 2018. The microbiome of Haemaphysalis

587 lemuris (Acari: Ixodidae), a possible vector of pathogens of endangered lemur species in

588 Madagascar. Ticks and Tick-borne Diseases 9:1252-1260.

589 Leclerque A. 2008. Whole genome-based assessment of the taxonomic position of the arthropod

590 pathogenic bacterium Rickettsiella grylli. FEMS Microbiology Letters 283(1):117-127.

591 Li JS, Chu F, Reilly A, Winslow GM. 2002. Antibodies Highly Effective in SCID Mice During

592 Infection by the Intracellular Bacterium Ehrlichia chaffeensis Are of Picomolar Affinity and

$593 \quad$ Exhibit Preferential Epitope and Isotype Utilization. The Journal of Immunology 169:1419-

$594 \quad 1425$.

595 Martin M. 2011. Cutadapt removes adapter sequences from high-throughput sequencing reads.

$596 \quad$ EMBnet.journal 17:10.

597 McMurdie PJ, Holmes S. 2013. Phyloseq: An R Package for Reproducible Interactive Analysis

$598 \quad$ and Graphics of Microbiome Census Data. PLoS ONE 8:e61217.

599 Murgia MV, Bell-Sakyi L, de la Fuente J, Kurtti TJ, Makepeace BL, Mans B, McCoy KD,

600 Munderloh U, Plantard O, Rispe C, Valle MR, Tabor A, Thangamani S, Thimmapuram J,

601 Hill CA. 2019. Meeting the challenge of tick-borne disease control: A proposal for 1000

602 Ixodes genomes. Ticks and Tick-borne Diseases 10:213-218.

603 Narasimhan S, Fikrig E. 2015. Tick microbiome: the force within. Trends in parasitology 
31:315-23.

605 Oksanen J, Blanchet FG, Friendly M, Kindt R, Legendre P, McGlinn D, Minchin PR, O'Hara

606 RB, Simpson GL, Solymos P, Stevens MHH, Szoecs E, Wagner H. 2019. vegan:

607 Community Ecology Package. R package version 2.5-2.

608 Paddock CD, Denison AM, Dryden MW, Noden BH, Lash RR, Abdelghani SS, Evans AE, Kelly

609 AR, Hecht JA, Karpathy SE, Ganta RR, Little SE. 2015. High prevalence of "Candidatus

610 Rickettsia andeanae"; and apparent exclusion of Rickettsia parkeri in adult Amblyomma

611 maculatum (Acari: Ixodidae) from Kansas and Oklahoma. Ticks and Tick-borne Diseases

$612 \quad 6: 297-302$.

613 Paddock CD, Lane RS, Staples JE, Labruna MB. 2016. Changing paradigms for tick-borne

614 diseases in the Americas. Page Global Health Impacts of Vector-Borne Diseases: Workshop

615 Summary.

616 Price MN, Dehal PS, Arkin AP. 2009. Fasttree: Computing large minimum evolution trees with

617 profiles instead of a distance matrix. Molecular Biology and Evolution 26:1641-1650.

618 Quast C, Pruesse E, Yilmaz P, Gerken J, Schweer T, Yarza P, Peplies J, Glöckner FO. 2013. The

619 SILVA ribosomal RNA gene database project: Improved data processing and web-based

620 tools. Nucleic Acids Research 41:D590-D596.

621 Regnery RL, Spruill CL, Plikaytis BD. 1991. Genotypic identification of rickettsiae and

622 estimation of intraspecies sequence divergence for portions of two rickettsial genes. Journal 623 of Bacteriology 173:1576-1589.

624 Rynkiewicz EC, Hemmerich C, Rusch DB, Fuqua C, Clay K. 2015. Concordance of bacterial 625 communities of two tick species and blood of their shared rodent host. Molecular Ecology $626 \quad 24: 2566-79$. 
627 Smith CCR, Snowberg LK, Gregory Caporaso J, Knight R, Bolnick DI. 2015a. Dietary input of 628 microbes and host genetic variation shape among-population differences in stickleback gut 629 microbiota. ISME Journal 9:2515-2526.

630 Smith TA, Driscoll T, Gillespie JJ, Raghavan R. 2015b. A Coxiella-like endosymbiontis a 631 potential vitamin source for the lone star tick. Genome Biology and Evolution 7(3):831-838. 632 Spor A, Koren O, Ley R. 2011. Unravelling the effects of the environment and host genotype on 633 the gut microbiome. Nature Reviews Microbiology 9(4):279-290.

634 Steury RA, Currey MC, Cresko WA, Bohannan BJM. 2019. Population genetic divergence and 635 environment influence the gut microbiome in oregon threespine stickleback. Genes $636 \quad \mathbf{1 0}(7): 484$.

637 Travanty NV, Ponnusamy L, Kakumanu ML, Nicholson WL, Apperson CS. 2019. Diversity and 638 structure of the bacterial microbiome of the American dog tick, Dermacentor variabilis, is 639 dominated by the endosymbiont Francisella. Symbiosis 79:239-250.

640 Trout Fryxell RT, DeBruyn JM. 2016. The microbiome of Ehrlichia-infected and uninfected 641 lone star ticks (Amblyomma americanum). PLoS ONE 11:e0146651.

642 Van Treuren W, Ponnusamy L, Brinkerhoff RJ, Gonzalez A, Parobek CM, Juliano JJ, Andreadis 643 TG, Falco RC, Beati Ziegler L, Hathaway N, Keeler C, Emch M, Bailey JA, Roe RM, 644 Apperson CS, Knight R, Meshnick SR. 2015. Variation in the Microbiota of Ixodes Ticks 645 with Regard to Geography, Species, and Sex. Applied and Environmental Microbiology $646 \quad$ 81(18):6200-6209.

647 Walters W, Hyde ER, Berg-Lyons D, Ackermann G, Humphrey G, Parada A, Gilbert JA, 648 Jansson JK, Caporaso JG, Fuhrman JA, Apprill A, Knight R. 2016. Improved Bacterial 16S 649 rRNA Gene (V4 and V4-5) and Fungal Internal Transcribed Spacer Marker Gene Primers 
650 for Microbial Community Surveys. mSystems 1(1):e0009-15.

651 Zhong J, Jasinskas A, Barbour AG. 2007. Antibiotic treatment of the tick vector Amblyomma 652 americanum reduced reproductive fitness. PLoS ONE 2(5):e405.

653

654 


\section{Table $\mathbf{1}$ (on next page)}

General information of Dermacentor variabilis samples used in this study. All individuals are from the USA. Each row corresponds to an individual tick specimen and the columns to the collection information. 
Table 1. General information of Dermacentor variabilis samples used in this study. All individuals are from the USA. Each row corresponds to an individual tick specimen and the columns to the collection information.

\begin{tabular}{|c|c|c|c|c|c|c|}
\hline RAW DATA ID & VOUCHER ID & COLLECTION EVENT ID & SEX & US STATE & LOCALITY & COORDINATES \\
\hline $119592-6$ & OSAL119943 & OSAL119592 & Female & IN & Hoosier National Forest & $38.52,-86.44$ \\
\hline $119592-7$ & OSAL119944 & OSAL119592 & Male & IN & Hoosier National Forest & $38.52,-86.44$ \\
\hline $119273-2$ & OSAL119553 & OSAL119273 & Female & IN & Splinter Ridge Wildlife area & $38.75,-85.20$ \\
\hline $119241-2$ & OSAL119260 & OSAL119241 & Female & $\mathrm{OH}$ & Battelle Darby Creek MP & $39.9,-83.21$ \\
\hline $119241-3$ & OSAL119261 & OSAL119241 & Male & $\mathrm{OH}$ & Battelle Darby Creek MP & $39.9,-83.21$ \\
\hline $119241-4$ & OSAL119262 & OSAL119241 & Male & $\mathrm{OH}$ & Battelle Darby Creek MP & $39.9,-83.21$ \\
\hline $119243-1$ & OSAL119267 & OSAL119243 & Female & $\mathrm{OH}$ & Glacier Ridge MP & $40.13,-83.18$ \\
\hline $119243-2$ & OSAL119268 & OSAL119243 & Female & $\mathrm{OH}$ & Glacier Ridge MP & $40.13,-83.18$ \\
\hline $119243-3$ & OSAL119269 & OSAL119243 & Male & $\mathrm{OH}$ & Glacier Ridge MP & $40.13,-83.18$ \\
\hline $119243-4$ & OSAL119270 & OSAL119243 & Male & $\mathrm{OH}$ & Glacier Ridge MP & $40.13,-83.18$ \\
\hline $119244-1$ & OSAL119400 & OSAL119244 & Female & $\mathrm{OH}$ & High banks MP & $40.15,-83.03$ \\
\hline 119244-4 & OSAL119403 & OSAL119244 & Male & $\mathrm{OH}$ & High banks MP & $40.15,-83.03$ \\
\hline $119247-1$ & OSAL119404 & OSAL119247 & Female & $\mathrm{OH}$ & Pickerington Ponds MP & $39.88,-82.79$ \\
\hline $119247-4$ & OSAL119407 & OSAL119247 & Male & $\mathrm{OH}$ & Pickerington Ponds MP & $39.88,-82.79$ \\
\hline $119248-1$ & OSAL119392 & OSAL119248 & Female & $\mathrm{OH}$ & Pickerington Ponds MP & $39.88,-82.80$ \\
\hline $119248-4$ & OSAL119395 & OSAL119248 & Male & $\mathrm{OH}$ & Pickerington Ponds MP & $39.88,-82.80$ \\
\hline $119250-2$ & OSAL119397 & OSAL119250 & Female & $\mathrm{OH}$ & Sharon Woods MP & $40.11,-82.95$ \\
\hline $119250-4$ & OSAL119399 & OSAL119250 & Male & $\mathrm{OH}$ & Sharon Woods MP & $40.11,-82.95$ \\
\hline $110559-1$ & OSAL110503 & OSAL110559 & Female & $\mathrm{OH}$ & Fernald Preserve & $39.29,-84.69$ \\
\hline 110559-2 & OSAL110504 & OSAL110559 & Female & $\mathrm{OH}$ & Fernald Preserve & $39.29,-84.69$ \\
\hline 119600B & OSAL129714 & OSAL119600 & Male & $\mathrm{OH}$ & Roads intersection & $39.13,-84.79$ \\
\hline $115093 A$ & OSAL129721 & OSAL115093 & Female & $\mathrm{OH}$ & Cuyahoga Valley & $41.289,-81.573$ \\
\hline 115093B & OSAL129722 & OSAL115093 & Female & $\mathrm{OH}$ & Cuyahoga Valley & $41.289,-81.573$ \\
\hline $115093 C$ & OSAL129723 & OSAL115093 & Female & $\mathrm{OH}$ & Cuyahoga Valley & $41.289,-81.573$ \\
\hline 115093D & OSAL129724 & OSAL115093 & Male & $\mathrm{OH}$ & Cuyahoga Valley & $41.289,-81.573$ \\
\hline $115093 E$ & OSAL129725 & OSAL115093 & Male & $\mathrm{OH}$ & Cuyahoga Valley & $41.289,-81.573$ \\
\hline 119928A & OSAL129691 & OSAL119928 & Female & $\mathrm{OH}$ & Strouds Run SP & $39.369,-82.042$ \\
\hline 119928B & OSAL129692 & OSAL119928 & Female & $\mathrm{OH}$ & Strouds Run SP & $39.369,-82.042$ \\
\hline $119928 C$ & OSAL129693 & OSAL119928 & Male & $\mathrm{OH}$ & Strouds Run SP & $39.369,-82.042$ \\
\hline 119928D & OSAL129694 & OSAL119928 & Male & $\mathrm{OH}$ & Strouds Run SP & $39.369,-82.042$ \\
\hline
\end{tabular}




\begin{tabular}{|c|c|c|c|c|c|c|}
\hline 119572A & OSAL129695 & OSAL119572 & Female & $\mathrm{OH}$ & Oak Openings MP & $41.549,-83.854$ \\
\hline 119572B & OSAL129696 & OSAL119572 & Female & $\mathrm{OH}$ & Oak Openings MP & $41.549,-83.854$ \\
\hline 119572C & OSAL129697 & OSAL119572 & Male & $\mathrm{OH}$ & Oak Openings MP & $41.549,-83.854$ \\
\hline 119572D & OSAL129698 & OSAL119572 & Male & $\mathrm{OH}$ & Oak Openings MP & $41.549,-83.854$ \\
\hline 119567A & OSAL129707 & OSAL119567 & Female & $\mathrm{TN}$ & Knoxville & $35.390,-84.226$ \\
\hline 119567B & OSAL129708 & OSAL119567 & Female & $\mathrm{TN}$ & Knoxville & $35.390,-84.226$ \\
\hline $119567 C$ & OSAL129709 & OSAL119567 & Female & $\mathrm{TN}$ & Knoxville & $35.390,-84.226$ \\
\hline 119567D & OSAL129710 & OSAL119567 & Male & TN & Knoxville & $35.390,-84.226$ \\
\hline 119567E & OSAL129712 & OSAL119567 & Male & $\mathrm{TN}$ & Knoxville & $35.390,-84.226$ \\
\hline N8805A & OSAL129702 & USNMENT01358805 & Male & OK & Washita Co. & $35.411,-99.059$ \\
\hline N8805B & OSAL129703 & USNMENT01358805 & Male & OK & Washita Co. & $35.411,-99.059$ \\
\hline N8805C & OSAL129704 & USNMENT01358805 & Male & OK & Washita Co. & $35.411,-99.059$ \\
\hline N8806A & OSAL129705 & USNMENT01358806 & Female & OK & Washita Co. & $35.411,-99.059$ \\
\hline N8806B & OSAL129706 & USNMENT01358806 & Female & OK & Washita Co. & $35.411,-99.059$ \\
\hline N8464B & OSAL129701 & USNMENT01358464 & Male & VA & Warren Co. & $38.893,-78.14$ \\
\hline 119951 & OSAL129711 & OSAL119951 & Female & GA & Statesboro & $32.42,-81.77$ \\
\hline 119952A & OSAL129715 & OSAL119952 & Female & GA & Statesboro & $32.42,-81.77$ \\
\hline 115086 & OSAL129836 & OSAL115086 & Male & MN & Carlos Avery & $45.287,-93.122$ \\
\hline $115087 A$ & OSAL129717 & OSAL115087 & Female & MN & Camp Ripley & $46.076,-94.349$ \\
\hline 115087B & OSAL129718 & OSAL115087 & Male & $\mathrm{MN}$ & Camp Ripley & $46.076,-94.349$ \\
\hline 119918A & OSAL129829 & OSAL119918 & Female & MN & Columbus & 45.31, -93.02 \\
\hline 119918B & OSAL129830 & OSAL119918 & Female & MN & Stutsman Co. & $47.23,-98.87$ \\
\hline N128168B & OSAL129979 & USNMENT01358520 & Male & ND & Stutsman Co. & $47.23,-98.87$ \\
\hline 115139A & OSAL129834 & OSAL115139 & Female & ME & Crescent Beach & $43.56,-70.23$ \\
\hline 115139B & OSAL129835 & OSAL115139 & Male & ME & Crescent Beach & $43.56,-70.23$ \\
\hline 115140 & OSAL129833 & OSAL115140 & Female & ME & Unknown & Unknown \\
\hline 119276-2 & OSAL129559 & OSAL119276 & Female & MD & Aberdeen Providing Ground & $39.46,-76.12$ \\
\hline $119276-3$ & OSAL129560 & OSAL119276 & Female & MD & Aberdeen Providing Ground & $39.46,-76.12$ \\
\hline $119276-5$ & OSAL129562 & OSAL119276 & Female & MD & Aberdeen Providing Ground & $39.46,-76.12$ \\
\hline 119276-6 & OSAL129563 & OSAL119276 & Female & MD & Aberdeen Providing Ground & $39.46,-76.12$ \\
\hline 115101 & OSAL129845 & OSAL115101 & Male & CA & Napa Co & $38.215,-122.33$ \\
\hline $115102 A$ & OSAL129846 & OSAL115102 & Male & $\mathrm{CA}$ & Lake Co. & $39.139,-122.886$ \\
\hline $115102 \mathrm{C}$ & OSAL129848 & OSAL115102 & Male & CA & Lake Co. & $39.139,-122.886$ \\
\hline 115105B & OSAL129852 & OSAL115105 & Female & WA & Whitman Co. & $46.623,-117.228$ \\
\hline
\end{tabular}




\section{Table 2 (on next page)}

Rickettsia spp. and Anaplasma spp. screening results divided by state. 
1 Table 2. Rickettsia spp. and Anaplasma spp. screening results divided by state.

\begin{tabular}{|c|c|c|c|c|c|c|}
\hline \multirow[b]{2}{*}{ State } & \multirow[b]{2}{*}{$\begin{array}{c}\text { Year of } \\
\text { collection }\end{array}$} & \multirow[b]{2}{*}{$\begin{array}{l}\text { Number of } \\
\text { ticks positive } \\
\text { for } \\
\text { rickettsiales }\end{array}$} & \multicolumn{4}{|c|}{ Total number of ticks positive } \\
\hline & & & R. rhipicephali & R. montanensis & R. bellii & Anaplasma spp \\
\hline California & 2017 & $2 / 3(66.7 \%)$ & & & $+(2)$ & \\
\hline Georgia & 2013,2016 & $1 / 2(50 \%)$ & & $+(1)$ & & \\
\hline Indiana & 2017 & $0 / 3$ & & & & \\
\hline Maryland & 2017 & $0 / 4$ & & & & \\
\hline Maine & 2017 & $0 / 3$ & & & & \\
\hline Minnesota & 2017 & $0 / 5$ & & & & \\
\hline North Dakota & 2017 & $1 / 1(100 \%)$ & & $+(1)$ & & \\
\hline Ohio & 2016, 2017 & $1 / 31(3.2 \%)$ & & $+(1)$ & & \\
\hline Oklahoma & 2017 & $3 / 5(60 \%)$ & $+(2)$ & & & $+(1)$ \\
\hline Tennessee & 2017 & $3 / 5(60 \%)$ & & $+(3)$ & & \\
\hline Virginia & 2017 & $0 / 1$ & & & & \\
\hline Washington & 2017 & $0 / 1$ & & & & \\
\hline Total & & $11 / 64(17.2 \%)$ & 2 & 6 & 2 & 1 \\
\hline
\end{tabular}


Figure 1

Map of the USA showing the sampling locations.

The size of the circles reflects the number of specimens analyzed from each locality, from 1 to 5 .

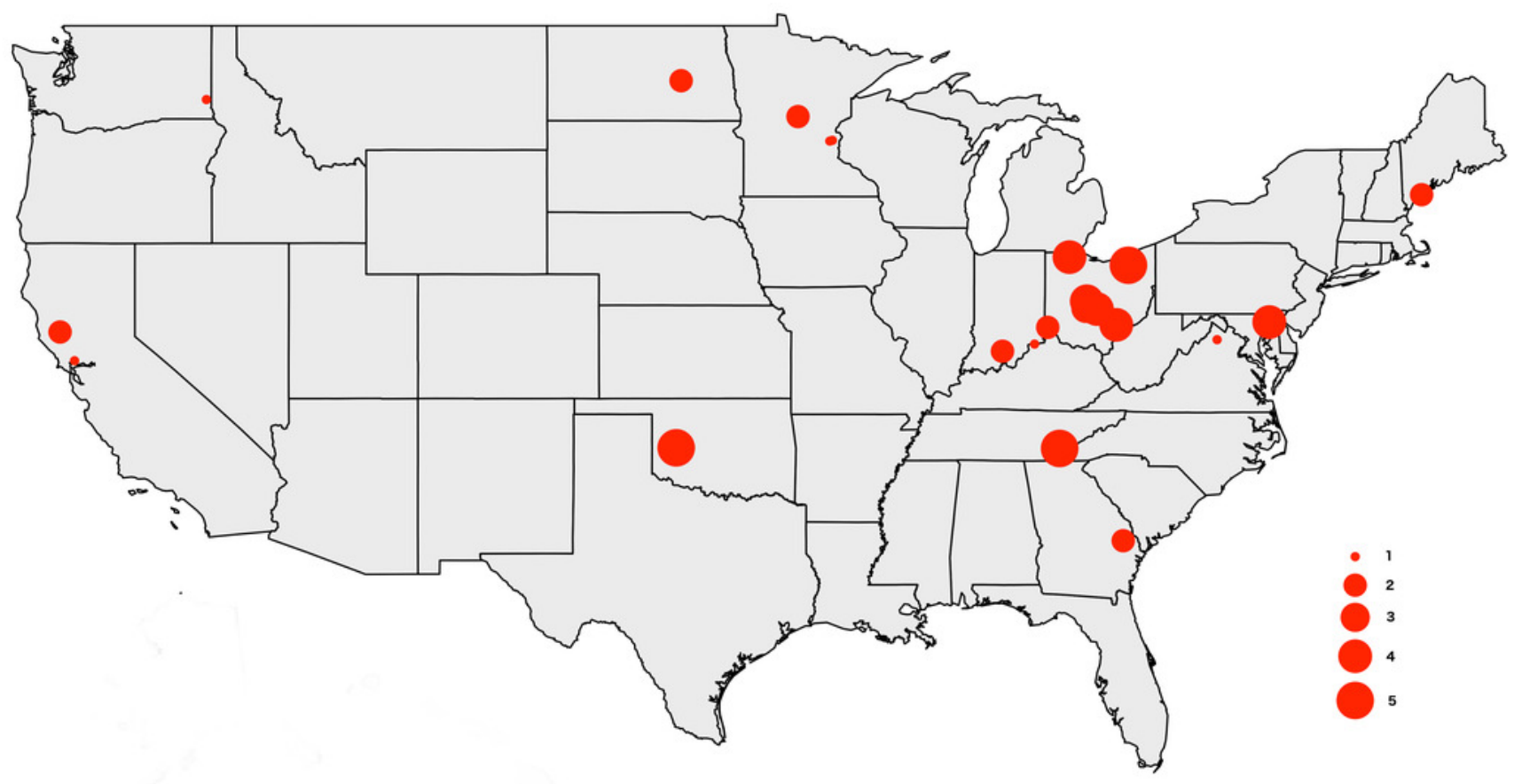


Figure 2

Barplots of the relative abundance of bacteria.

'(A) Barplot of the relative abundance of bacteria at the Order level. The $x$ axis corresponds to individual tick specimens, and the $y$ axis shows the relative abundance of the different bacteria Orders. Bacteria orders are color-coded as shown below the plot; (B) Similar to A), barplot of the relative abundance of the bacteria at the genus level.'
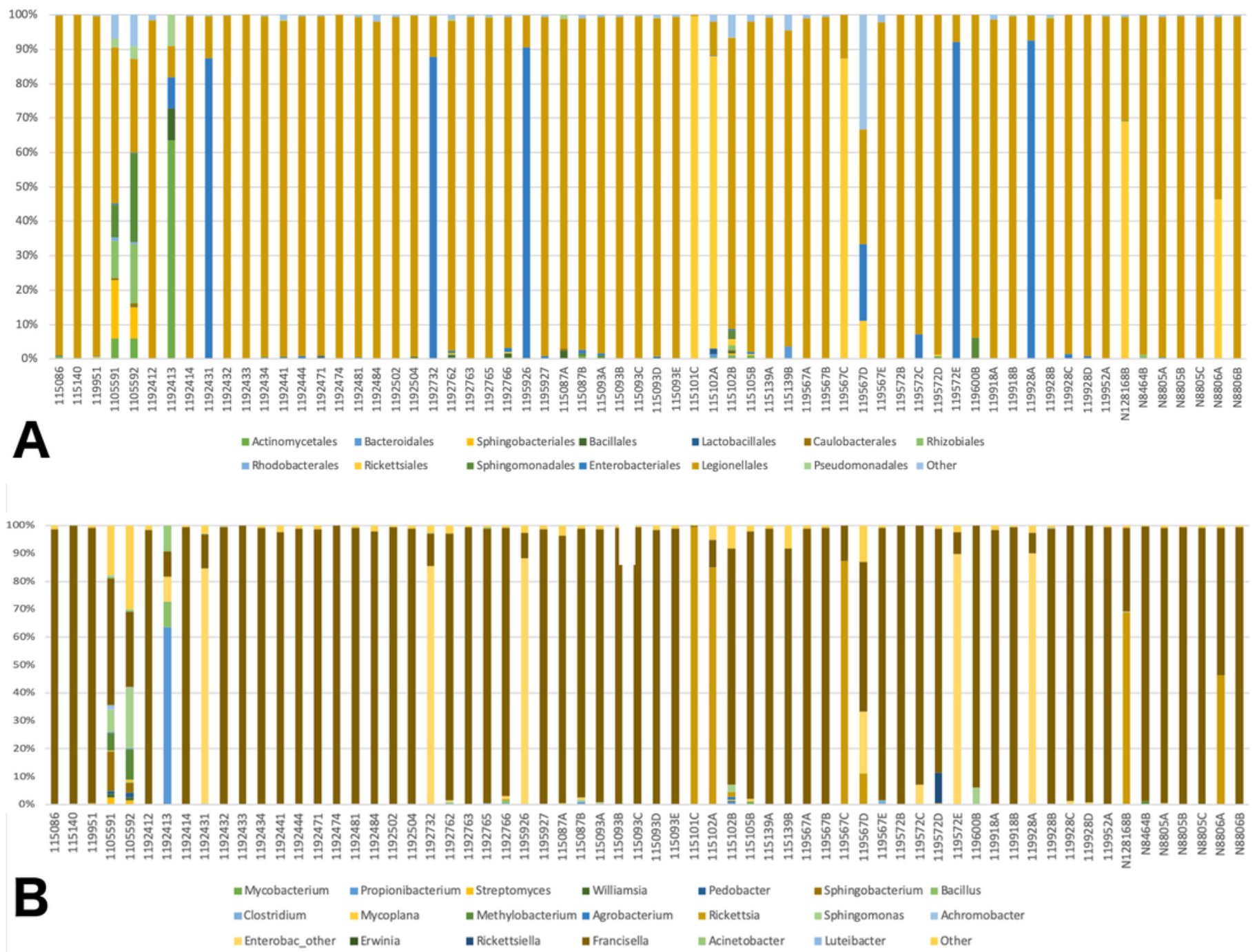
Figure 3

Dendrogram showing the relationship between all samples, and the different groupings.

Groupings names: Eastern, Western, CA (samples from California), WA (samples from

Washington), and $\mathrm{OH}$ Hamilton Co (samples collected in Hamilton County, Ohio).

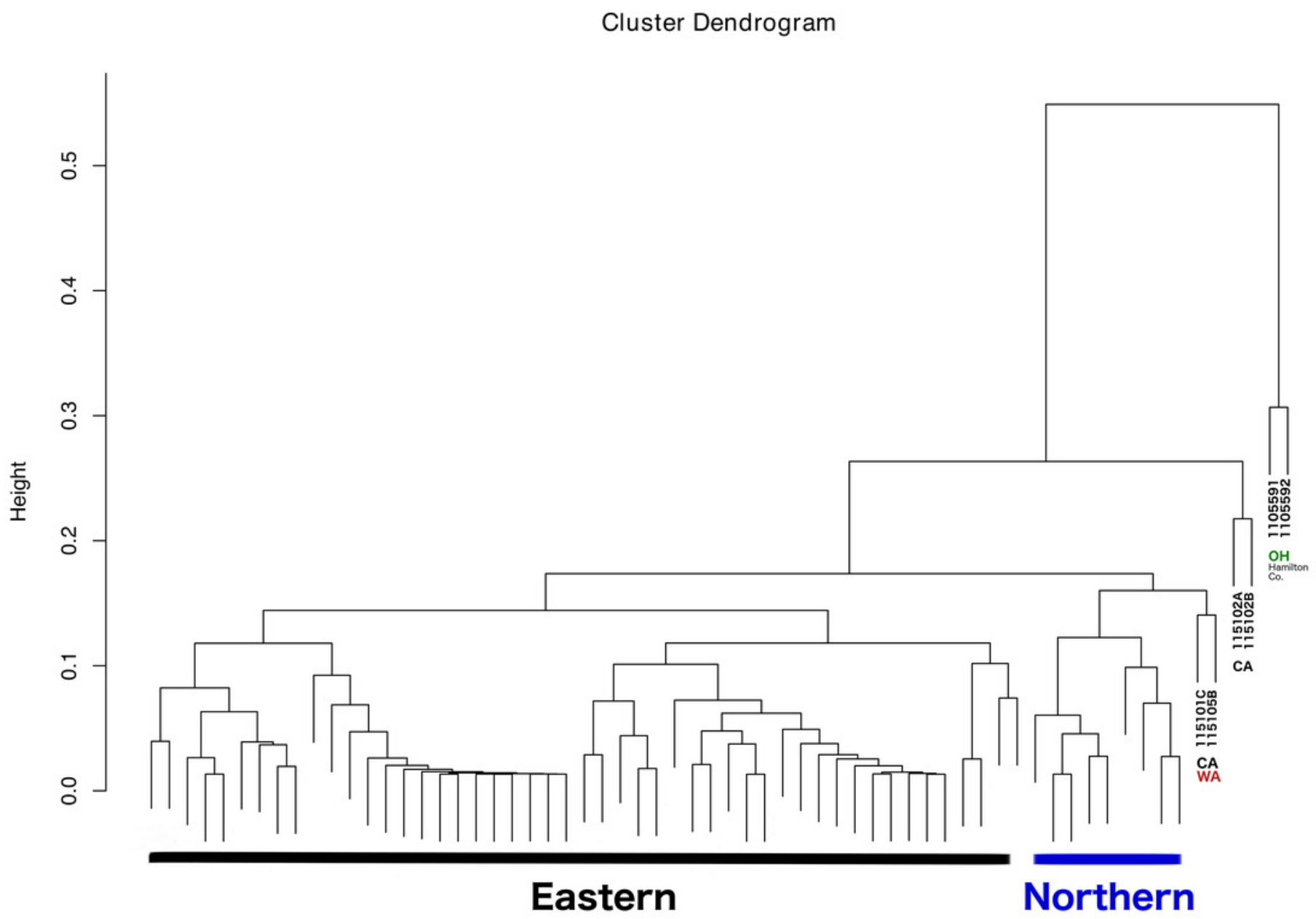


Figure 4

Non-metric multidimensional scaling and Principal coordinates analyses.

'(A) Non metric multidimensional scaling analysis of the samples. Different colors represent the different genetic clusters: Eastern (red), Northern (green), and Western (blue). (B) Principal coordinates analysis of the samples. Different colors represent the different genetic clusters: Eastern (blue), Northern (orange), and Western (black). Axis 1 explains $74.9 \%$ of the variance, and Axis 2 explains $10 \%$ of it.'

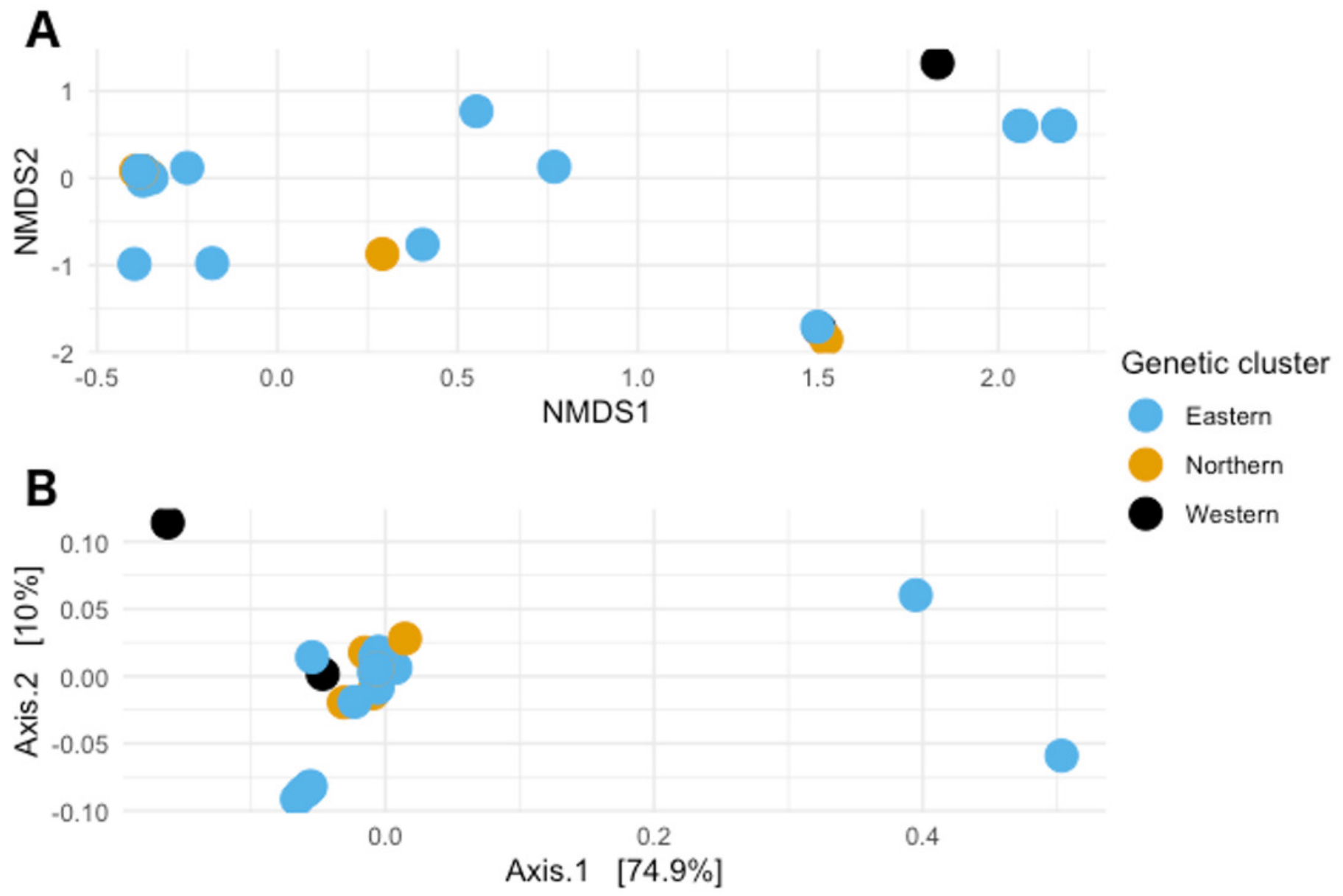


Figure 5

Average abundance of bacteria genera by genetic cluster.

Average abundance (y axis) of bacteria genera ( $x$ axis) by genetic clusters (Northern, Eastern, Western), and sex (male in orange, female in blue).

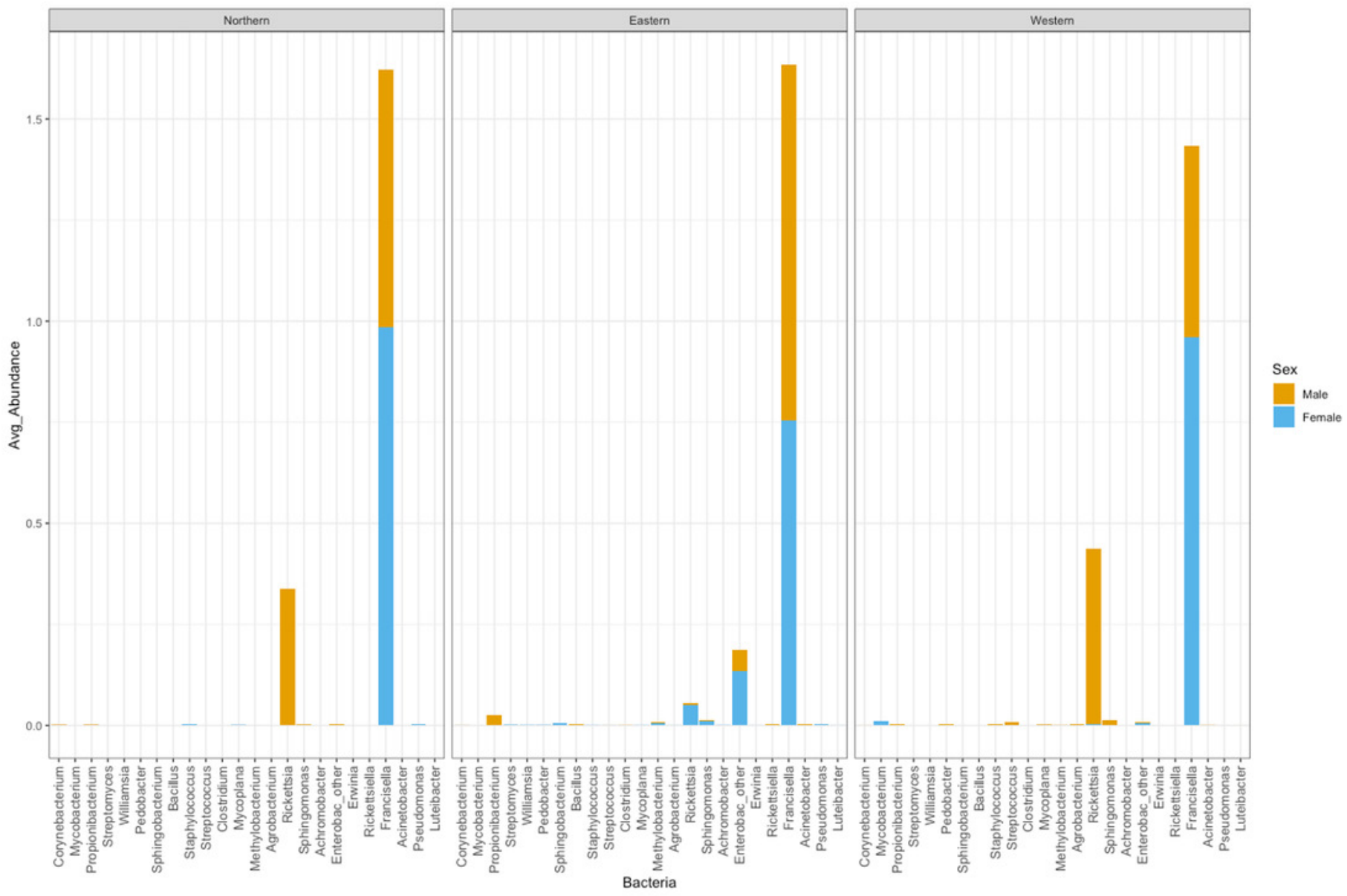




\section{Figure 6}

Pie chart plots of proportions of infection by genetic clusters.

Pie chart plots of proportions of infection by genetic clusters. Each plot corresponds to an agent; A) R. rhipicephali, B) R. montanensis, C) R. bellii, D) Anaplasma spp. The colors represent the genetic clusters (Northern in black; Eastern in orange; and Western in blue). 


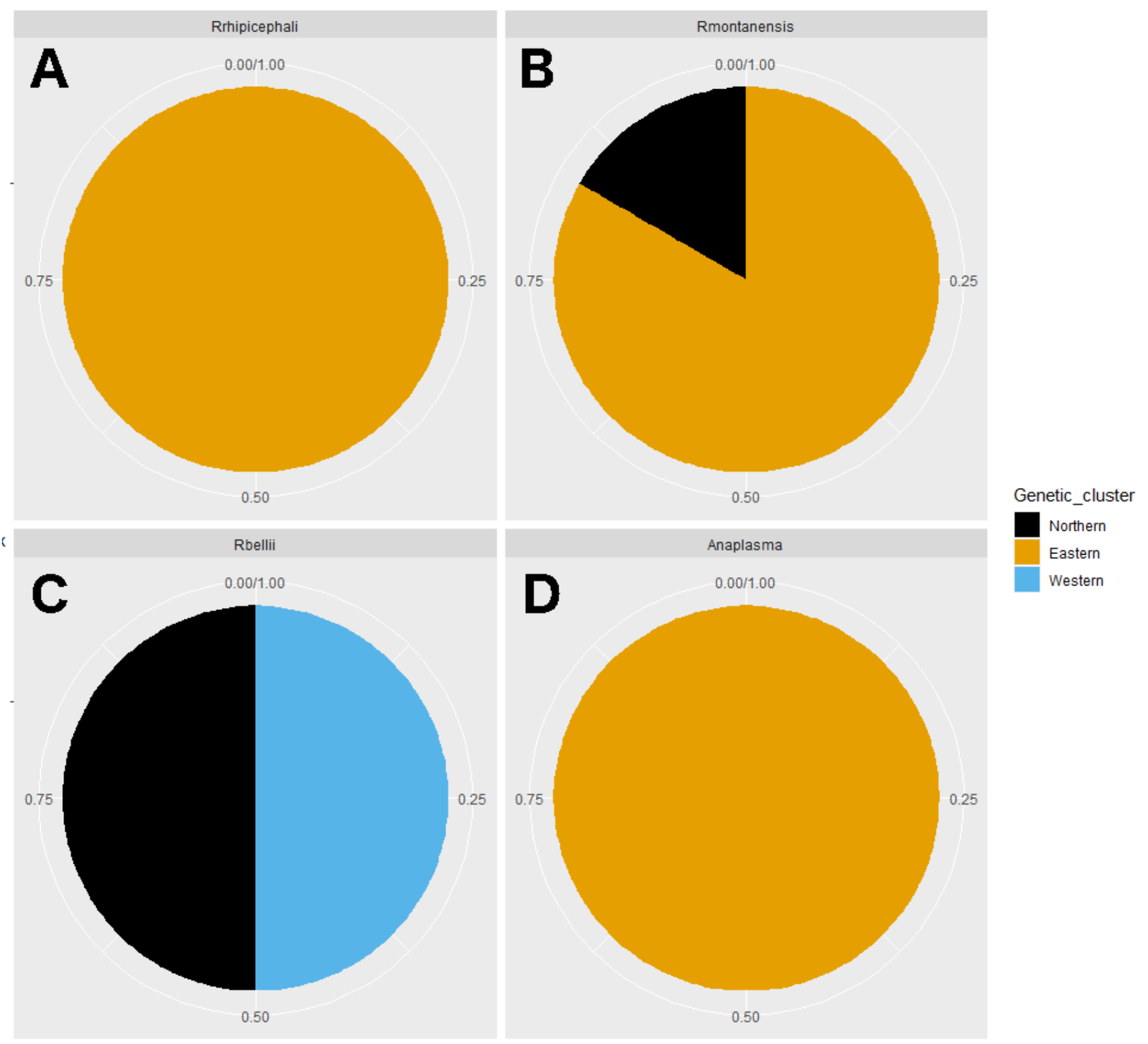

Proportion_of_Infection 